\title{
Estudio Morfométrico del Radio Proximal en una Población Chilena
}

\author{
Morphometric Study of the Proximal Radius in a Chilean Population
}

Francisco J. Muñoz ${ }^{1,3}$; Patricio A. Alfaro ${ }^{1,3}$; Felipe Carcamo ${ }^{1}$;

Pedro I. Reyes ${ }^{2}$; Fabian E. Padilla ${ }^{1,3}$ \& Edgardo S. Sanzana ${ }^{1,3}$

\begin{abstract}
MUÑoz, F. J.; AlFARO, P. A.; CARCAMO, F.; REYES, P. I.; PADILLA, F. E. \& SANZANA, E. S. Estudio morfométrico del radio proximal en una población chilena. Int. J. Morphol., 39(2):484-488, 2021.

RESUMEN: El objetivo de esta investigación fue determinar la morfometría del extremo proximal del radio, mediante mediciones efectuadas en la cabeza, cuello y tuberosidad del radio, en una población chilena y compararlas según sexo. Se efectuó un estudio transversal analizando exámenes de Tomografía Computarizada (TC) de codo, realizados entre enero de 2014 y diciembre de 2018 . Se incluyó 32 TC, correspondientes a 16 hombres y 16 mujeres. Se transfirió las imágenes formateadas al software RadiAnt, para efectuar las mediciones en el radio proximal. El análisis estadístico de los resultados se realizó mediante el software SPSS 22 . El diámetro de la

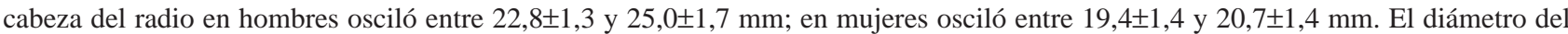

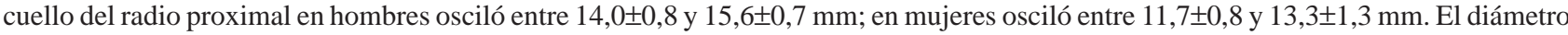

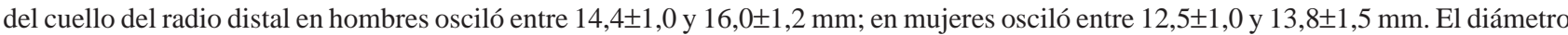

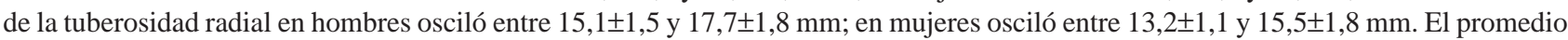
de altura de la cabeza del radio fue de 11,2 $\pm 1,2 \mathrm{~mm}$ en hombres y de 9,5+0,8 mm en mujeres. El análisis comparativo entre sexos mostró diferencias estadísticamente significativas en todas las mediciones precedentes. El promedio de altura del cuello del radio fue de $11,2 \pm 1,2$ $\mathrm{mm}$ en hombres y $10,1 \pm 1,6 \mathrm{~mm}$ en mujeres, sin diferencia significativa $(\mathrm{p}=0,15)$. Los valores promedios de la morfometría del radio proximal de la población chilena difieren de los descritos para la población europea y presentan algunas similitudes con la población china. Nuestros resultados pueden ser de utilidad para el diseño de implantes y prótesis para el extremo proximal del radio y para una correcta planificación quirúrgica en ortopedia y traumatología.
\end{abstract}

PALABRAS CLAVE: Radio proximal; Cabeza del radio; Cuello del radio; Tuberosidad radial; Tomografía computarizada

\section{INTRODUCCIÓN}

La anatomía de la articulación del codo es compleja. Las superficies de la articulación húmeroradial son congruentes y contribuyen a la estabilidad ósea del codo (Bryce \& Armstrong, 2008). La cabeza del radio se comporta como estabilizadora primaria del estrés longitudinal y secundaria del estrés en valgo del codo. Cada uno de los estabilizadores estáticos y dinámicos del codo sirven para mantener las superficies de las articulaciones en posición congruente. (Johnson \& King, 2005). La contribución relativa de estas estructuras a la estabilidad varía en función de la orientación articular y el grado de activación muscular (Morrey, 2008).

La cabeza del radio no es perfectamente circular y se equilibra de manera variable desde el eje del cuello del radio. Morfológicamente la cabeza del radio tiene una forma elíptica en un $60 \%$ de los casos, con un diámetro promedio de $22 \mathrm{~mm}$. En un $40 \%$ posee forma circular con un diámetro promedio de $21 \mathrm{~mm}$. Estas variaciones deben ser consideradas en el manejo quirúrgico de las lesiones de la cabeza del radio (Beredjiklian et al., 1999; King et al., 2001).

La morfología de la cabeza del radio es clínicamente relevante para las diversas opciones de tratamientos quirúrgicos vigentes, que incluyen reducción abierta y fijación interna, cupulectomía o artroplastía de sustitución (King et al., 1991; Charalambous et al., 2011). La artroplastía es la opción terapéutica de elección en los casos de fracturas multifragmentarias del radio proximal. La endoprótesis de la cabeza del radio más usada es metálica, modular y no cementada. Para su óptimo diseño se requiere conocer los

\footnotetext{
${ }^{1}$ Departamento de Cirugía. Facultad de Medicina, Universidad de Concepción, Chile.

${ }^{2}$ Departamento de Especialidades, Facultad de Medicina, Universidad de Concepción, Chile.

${ }^{3}$ Servicio de Ortopedia y Traumatología, Hospital Traumatológico, Concepción, Chile.
} 
parámetros anatómicos de la población objetivo (Ring et al., 2002; Rosenblatt et al., 2008; Ring, 2011).

Diversos métodos han sido empleados para evaluar la morfología y morfometría del radio proximal, que incluyen radiología convencional, tomografía axial computada (TC), resonancia nuclear magnética (RNM) y diversos calibradores (Kuhn et al., 2012). No hemos encontrado estudios anatómicos que cuantifiquen las dimensiones de las estructuras del radio proximal en nuestra población nacional.

El objetivo de esta investigación fue determinar la morfometría del extremo proximal del radio, mediante mediciones efectuadas en la cabeza del radio, cuello y tuberosidad radial, en una población chilena y compararlas según sexo.

\section{MATERIAL Y MÉTODO}

Se realizó un estudio transversal evaluando los exámenes de TC realizados en nuestro centro, en el periodo entre enero de 2014 y diciembre del 2018, disponibles en la base de datos imagenológicos Synapse. Esta investigación fue aprobada por el Comité Ético Científico del Servicio de Salud Concepción, Chile (Código CEC: 19-03-13).

Se analizó 32 exámenes de TC del radio proximal, de 16 pacientes de sexo masculino y 16 de sexo femenino. El promedio de edad de los casos fue de 37,3 años (rango: 15 72 años). Se excluyeron los pacientes que presentaban fractura de la cabeza del radio, imagen deficiente del segmento en estudio o presencia de material de osteosíntesis en el radio proximal. Se realizó un muestreo consecutivo de casos, anali-

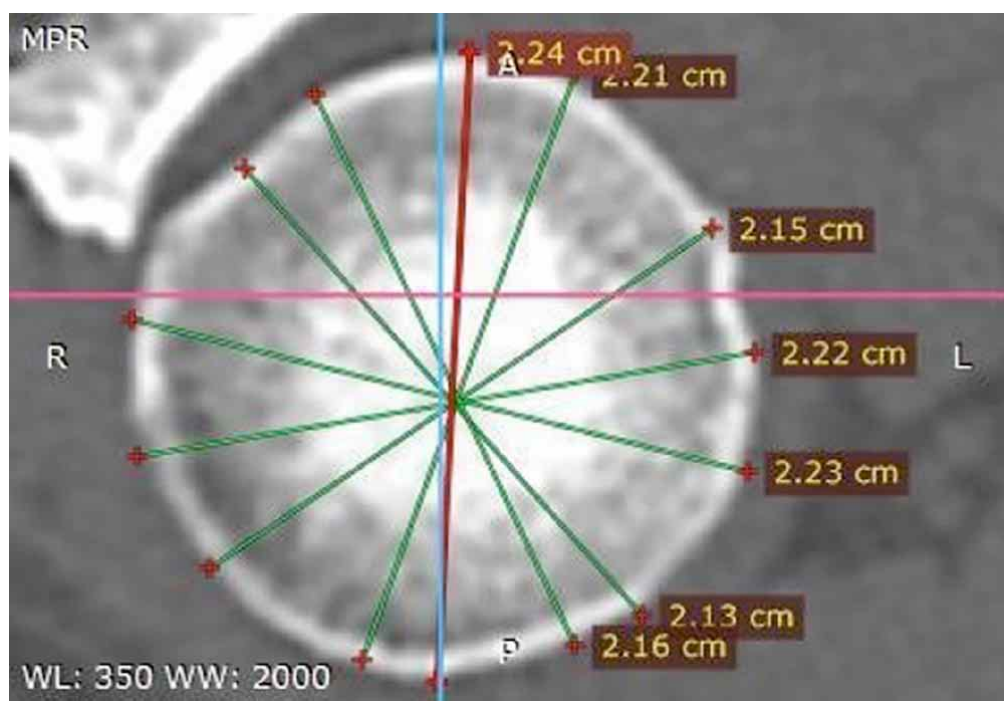

Fig. 1. Medición del diámetro de la cabeza del radio en el plano transversal. zando los exámenes de TC realizados en el periodo de estudio, con el tomógrafo modelo Toshiba Aquilion 64 ®.

Las imágenes se transfirieron formateadas de forma multiplanar al software RadiAnt, que fue utilizado para realizar el análisis y las mediciones de la morfología ósea del radio proximal. Las mediciones fueron obtenidas por dos evaluadores de manera independiente, uno de ellos radiólogo músculo-esquelético. Las mediciones morfológicas estudiadas correspondieron al diámetro de la cabeza del radio, el diámetro del cuello del radio proximal y distal y el diámetro de la tuberosidad radial, registrando los valores mínimos y máximos de todas ellas en el plano transversal. Accesoriamente, en el plano sagital se midió la altura de la cabeza del radio y del cuello del radio, registrando un valor único. Las mediciones obtenidas fueron expresadas en milímetros ( $\mathrm{mm})$.

Se seleccionaron las mediciones propuestas en estudios similares anteriores (Johnson \& King; Rosenblatt et al.; Kuhn et al.). Para la medición del diámetro de la cabeza del radio se identificó en un corte transversal un segmento donde la estructura ósea tenía su mayor homogeneidad y densidad. Se trazaron múltiples mediciones en línea recta, todas las cuales pasaban por un mismo punto central, seleccionando aquellas que reflejaban el valor del diámetro mínimo y el valor del diámetro máximo (Fig. 1).

Las medidas diametrales de la porción proximal del cuello del radio se obtuvieron en el segmento más estrecho, con márgenes redondeados, homogéneos y densos (Fig. 2A). Para la porción distal del cuello del radio se utilizó el corte previo al inicio de la tuberosidad radial (Fig. 2B). Para ambas mediciones se trazaron múltiples líneas, pasando por un punto central y se registró el valor mínimo y máximo para las dos secciones mencionadas (Figs. 2A,B).

Para la medición del diámetro de la tuberosidad radial se utilizó el segmento donde se observaba el área de mayor diámetro (Fig. 3). Al igual que en los cortes anteriores se realizaron múltiples trazados pasando por un punto central, registrando el diámetro mínimo y máximo obtenidos.

El análisis estadístico se realizó mediante el software SPSS IBM para Windows (Versión 22, IBM Corp.), empleando el Test U de Mann-Whitney para la comparación entre hombres y mujeres, de las mediciones promedios obtenidas en la cabeza del radio, cuello del radio y tuberosidad radial. 

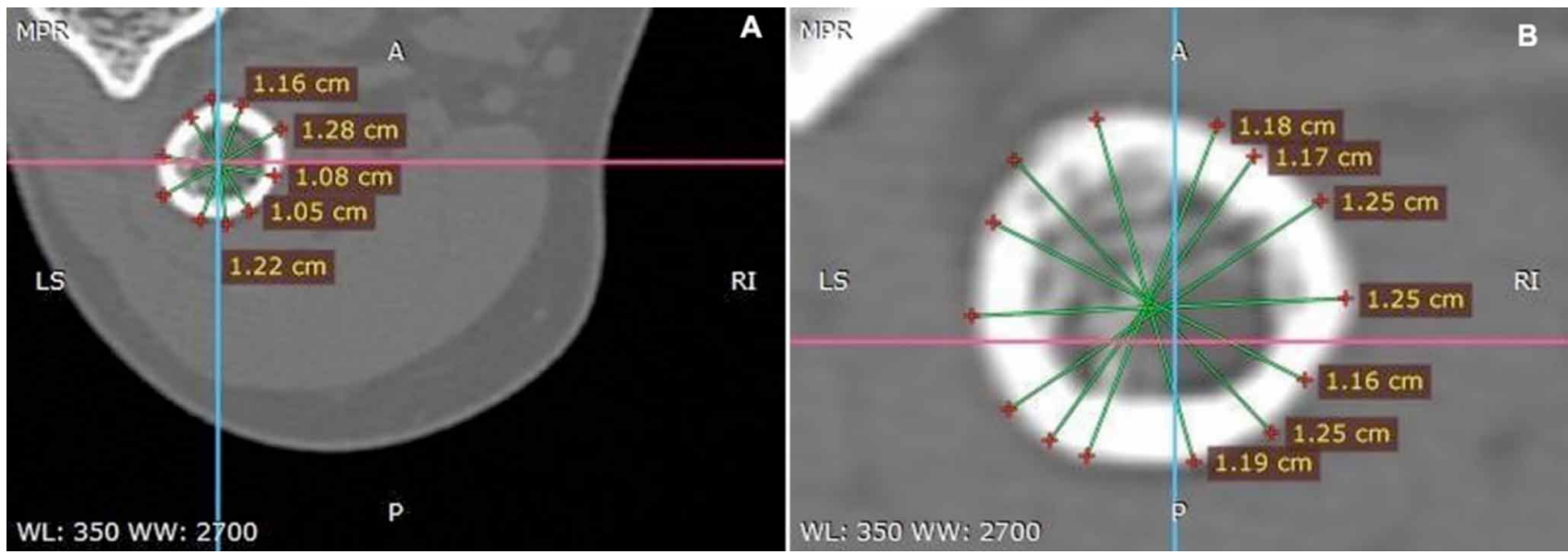

Fig. 2. A. Medición del diámetro del cuello del radio proximal en el plano transversal. B. Medición del diámetro del cuello del radio distal en el plano transversal.

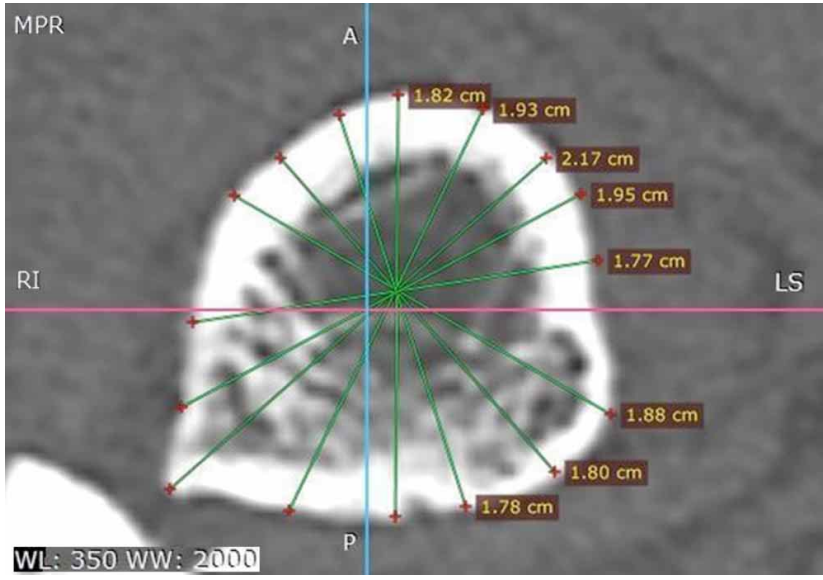

Fig. 3. Medición del diámetro de la tuberosidad radial en el plano transversal.

\section{RESULTADOS}

Parámetros morfológicos del radio proximal según sexo, obtenidos en una población chilena representada por 32 pacientes, 16 hombres y 16 mujeres se presentan en Tabla I.

Valores en el plano transversal: En la cabeza del radio de los hombres la diferencia entre el diámetro máximo y mínimo fue de 2,2 $\pm 0,4 \mathrm{~mm}$ y en las mujeres fue de $1,7 \mathrm{~mm}$. La diferencia entre los promedios mínimos y máximos según sexo fueron significativos $(\mathrm{p}<0,05)$.

En el cuello del radio proximal de los hombres la diferencia entre el diámetro máximo y mínimo fue de $1,6 \mathrm{~mm}$ y en las mujeres fue de $1,6 \pm 0,5 \mathrm{~mm}$. chilena.
En el cuello del radio distal, correspondiente a la unión cuello-tuberosidad, la diferencia en los hombres entre el diámetro máximo y mínimo fue de 1,6 $\pm 0,2 \mathrm{~mm}$ y en las mujeres fue de $1,3 \pm 0,5 \mathrm{~mm}$.

En la tuberosidad radial de los hombres la diferencia entre el diámetro máximo y mínimo fue de $2,6 \pm 0,3 \mathrm{~mm}$ y en las mujeres fue de $2,3 \pm 0,7 \mathrm{~mm}$.

La diferencia entre los promedios mínimos y máximos de todos los parámetros previamente señalados, entre hombres y mujeres, fueron estadísticamente significativas $(\mathrm{p}<0,05)$.

Valores en el plano sagital: La cabeza del radio mostró una altura promedio de $11,2 \pm 1,2 \mathrm{~mm}$ en hombres y de $9,5 \pm 0,8 \mathrm{~mm}$ en mujeres. La diferencia entre la altura promedio de hombres y mujeres fue estadísticamente significativa $(\mathrm{p}<0,05)$.

Tabla I. Parámetros morfológicos del radio proximal según sexo en una población

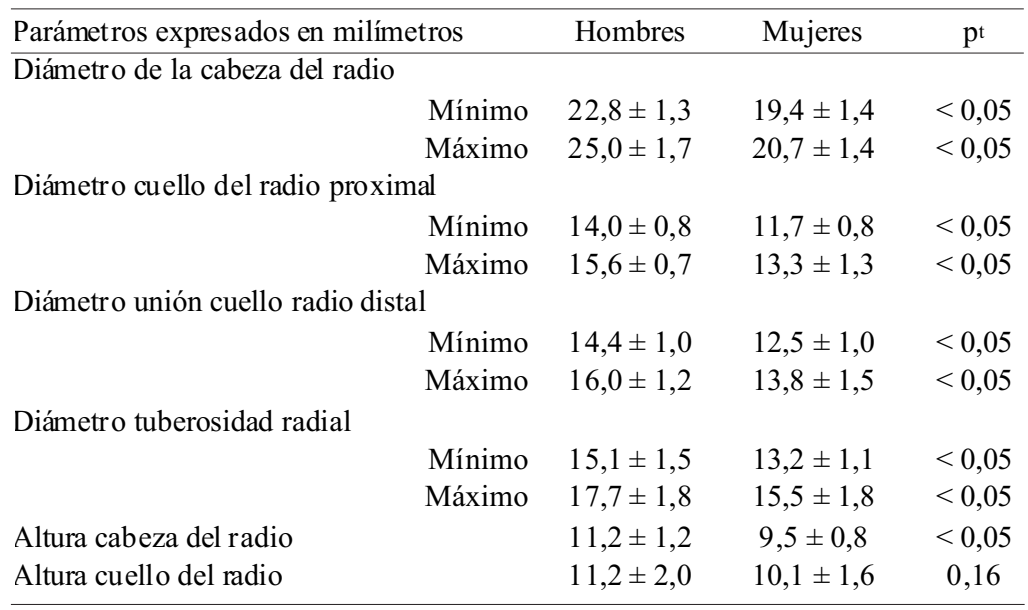


MUÑOZ, F. J.; ALFARO, P. A.; CARCAMO, F.; REYES, P. I.; PADILLA, F. E. \& SANZANA, E. S. Estudio morfométrico del radio proximal en una población chilena Int. J. Morphol., 39(2):484-488, 2021.

El cuello del radio mostró una altura promedio de $11,2 \pm 2,0 \mathrm{~mm}$ en hombres y 10,1 $\pm 1,6 \mathrm{~mm}$ en mujeres. Esta diferencia no era estadísticamente significativa $(\mathrm{p}=0,16)$

\section{DISCUSIÓN}

Diferentes métodos y técnicas han sido utilizados para estudiar la morfología del radio proximal, siendo de empleo más habitual la radiología estándar, la TC, la RNM y diversos tipos de calibradores (Cone et al., 1983; Captier et al., 2002; Roidis et al., 2003). Dado que no encontramos datos de referencia publicados previamente sobre la morfología y morfometría del radio proximal en la población chilena, nos propusimos obtener esta información desde nuestra base de datos imagenológicos institucional.

El estudio morfométrico del extremo proximal del radio nos ha permitido obtener valores promedio, que son comparativamente diferentes a los descritos previamente para la población europea (Swieszkowski et al., 2001; Popovic et al., 2005; Koslowsky et al., 2007). En cambio, la altura de la cabeza del radio reportada en nuestro estudio es similar a la informada en un estudio de población china (Kang et al., 2015), resultado que nos permite establecer la existencia de algunas similitudes antropométricas con nuestra población. De esta manera, dispositivos de osteosíntesis o protésicos para el extremo proximal del radio, diseñados para la población china, deberían presentar una mejor correlación anatómica para nuestra población.

La ausencia de información de referencia sobre la morfometría del radio proximal en la población chilena, nos permiten considerar que los datos reportados en este estudio pueden ser relevantes para la planificación quirúrgica, para la elección de implantes y para el diseño de prótesis de radio proximal que puedan ser solicitadas a medida (custom made) para nuestros pacientes (Gupta et al., 2015).

Dentro de las limitaciones de este estudio se encuentra la ausencia de estandarización en la adquisición de la imagen, hecho que carece de inconvenientes dado que el software empleado para realizar las mediciones permite rotar las imágenes en todos los sentidos requeridos. La realización del estudio en un solo centro hospitalario, disminuye la posibilidad de variación existente entre las poblaciones de un mismo país (Gupta et al., 1997).

La TC es un método imagenológico de uso frecuente en la clínica y de elección para el análisis de la anatomía ósea. Las imágenes obtenidas para efectuar las mediciones fueron realizadas con el mismo tomógrafo, disminuyendo así la variabilidad en las mediciones debido a diferentes calidades imagenológicas. Todas las mediciones de nuestro estudio fueron realizadas independientemente por dos evaluadores, hecho que reduce las posibles distorsiones (Itamura et al., 2008).

La relativa uniformidad antropométrica de la población chilena, hace que los resultados obtenidos en esta investigación, puedan ser extrapolables a todos los habitantes de nuestro país (Llorca-Jaña et al., 2018). Asimismo, los datos morfométricos del extremo proximal del radio obtenidos en este estudio, pueden ser de utilidad para otras poblaciones sudamericanas, especialmente de las naciones del pacífico.

Coincidimos con estudios equivalentes, en el reporte de diferencias estadísticamente significativas en la morfometría del radio proximal entre la población masculina y femenina, información que debe ser considerada al momento de programar una intervención quirúrgica (Beredjiklian et al.; Rosenblatt et al.; Kang et al.).

En conclusión, la información cuantitativa obtenida mediante TC del extremo proximal del radio, es de gran valor para la caracterización antropométrica de poblaciones específicas. Los resultados de esta investigación pueden ser de utilidad para el diseño de implantes y prótesis para el radio proximal de nuestra población y para una correcta planificación quirúrgica en ortopedia y traumatología.

MUÑOZ, F. J.; ALFARO, P. A.; CARCAMO, F.; REYES, P. I.; PADILLA, F. E. \& SANZANA, E. S. Morphometric study of the proximal radius in a Chilean population. Int. J. Morphol., 39(2):484-488, 2021.

SUMMARY: The aim of this research was to determine the morphometry of the proximal radius in a Chilean population, by means of measurements made in head of radius, neck of radius and radial tuberosity, and to compare them according to sex. A cross-sectional study was conducted analyzing Computed Tomography scans (CT) of elbows, performed between January 2014 and December 2018. Thirty-two CT corresponding to 16 men and 16 women were included. The formatted images were transferred to the RadiAnt software in order to perform measurements in the proximal radius. The statistical analysis of the results was performed using the SPSS 22 software. The diameter of the head of radius in men ranged between $22.8 \pm 1.3$ and $25.0 \pm 1.7$ $\mathrm{mm}$; in women it ranged between $19.4 \pm 1.4$ and $20.7 \pm 1.4 \mathrm{~mm}$. The diameter of the proximal neck of radius in men ranged between $14.0 \pm 0.8$ and $15.6 \pm 0.7 \mathrm{~mm}$; in women, it ranged between $11.7 \pm 0.8$ and $13.3 \pm 1.3 \mathrm{~mm}$. The diameter of the distal neck of radius in men ranged between $14.4 \pm 1.0$ and $16.0 \pm 1.2 \mathrm{~mm}$; in women, it ranged between $12.5 \pm 1.0$ and $13.8 \pm 1.5 \mathrm{~mm}$. The diameter of radial tuberosty in men ranged between $15.1 \pm 1.5$ and $17.7 \pm 1.8 \mathrm{~mm}$; in women, it ranged between $13.2 \pm 1.1$ and $15.5 \pm 1.8 \mathrm{~mm}$. The mean 
height of the head of radius was $11.2 \pm 1.2 \mathrm{~mm}$ in men and $9.5 \pm 0.8$ $\mathrm{mm}$ in women. Statistically significant sex differences were revealed in all the preceding measurements. The mean height of the neck of radius was $11.2 \pm 1.2 \mathrm{~mm}$ in men and $10.1 \pm 1.6 \mathrm{~mm}$ in women, with no significant difference $(\mathrm{p}=0.15)$. The average values of morphometry of the proximal radius of the Chilean population differ from those describing the European population, and show some similarities with the Chinese population. Our results may be useful to design of implants and prostheses for the proximal radius, and to correct surgical planning in orthopedics and traumatology.

KEY WORDS: Proximal radius; Head of of radius; Neck of radius; Radial tuberosity; Computed tomography.

\section{REFERENCIAS BIBLIOGRÁFICAS}

Beredjiklian, P. K.; Nalbantoglu, U.; Potter, H. G. \& Hotchkiss, R. N. Prosthetic radial head components and proximal radial morphology: a mismatch. J. Shoulder Elbow. Surg., 8(5):471-5, 1999.

Bryce, C. D. \& Armstrong, A. D. Anatomy and biomechanics of the elbow. Orthop. Clin. North Am., 39(2):141-54, 2008.

Captier, G.; Canovas, F.; Mercier, N.; Thomas, E. \& Bonnel, F. Biometry of the radial head: biomechanical implications in pronation and supination. Surg. Radiol. Anat., 24(5):295-301, 2002.

Charalambous, C. P.; Stanley, J. K.; Mills, S. P.; Hayton, M. J.; Hearnden, A.; Trail, I. \& Gagey, O. Comminuted radial head fractures: aspects of current management. J. Shoulder Elbow Surg., 20(6):996-1007, 2011.

Cone, R. O.; Szabo, R.; Resnick, D.; Gelberman, R.; Taleisnik, J. \& Gilula, L. A. Computed tomography of the normal radioulnar joints. Invest. Radiol., 18(6):541-5, 1983.

Gupta, C.; Kalthur, S. G.; Malsawmzuali, J. C. \& D'Souza, A. S. A morphological and morphometric study of proximal and distal ends of dry radii with its clinical implications. Biomed. J., 38(4):323-8, 2015.

Gupta, G. G.; Lucas, G. \& Hahn, D. L. Biomechanical and computer analysis of radial head prostheses. J. Shoulder Elbow Surg., 6(1):37-48, 1997.

Itamura, J. M.; Roidis, N. T.; Chong, A. K.; Vaishnav, S.; Papadakis, S. A. \& Zalavras, C. Computed tomography study of radial head morphology. J. Shoulder Elbow Surg., 17(2):347-54, 2008.

Johnson, J. A. \& King, G. J. Anatomy and Biomechanics of the Elbow. In: Williams, G. R.; Yamaguchi, K.; Ramsey, M. L. \& Galatz, L. M. (Eds.). Shoulder and Elbow Arthroplasty. Philadelphia, Lippincott Williams and Wilkins, 2005. pp.279-96.

Kang, S. H.; Kim, D. Y.; Park, H. \& Song, H. S. Anthropometric study of the proximal radius: does radial head implant fit in all cases? Int. Orthop., 39(8):1553-61, 2015.

King, G. J.; Evans, D. C. \& Kellam, J. F. Open reduction and internal fixation of radial head fractures. J. Orthop. Trauma, 5(1):21-8, 1991.

King, G. J.; Zarzour, Z. D.; Patterson, S. D. \& Johnson, J. A. An anthropometric study of the radial head: implications in the design of a prosthesis. J. Arthroplasty, 16(1):112-6, 2001.

Koslowsky, T. C.; Beyer, F.; Germund, I.; Mader, K.; Jergas, M. \& Koebke, J. Morphometric parameters of the radial neck: an anatomical study. Surg. Radiol. Anat., 29(4):279-84, 2007.

Kuhn, S.; Burkhart, K. J.; Schneider, J.; Muelbert, B. K.; Hartmann, F.; Mueller, L. P. \& Rommens, P. M. The anatomy of the proximal radius: implications on fracture implant design. J. Shoulder Elbow Surg., 21(9):1247-54, 2012.

Llorca-Jaña, M.; Araya, R. \& Navarrete-Montalvo, J. Antropometría histórica de Chile: evolución de la estatura de la población en el largo plazo, siglos XVIII-XX. Estud. Atacam., 60:161-91, 2018.

Morrey, B. F. Anatomy of the Elbow Joint. In: Morrey, B. F. (Ed.). The
Elbow and its Disorders. Philadelphia, Saunders, 2008. pp.11-39.

Popovic, N.; Djekic, J.; Lemaire, R. \& Gillet, P. A comparative study between proximal radial morphology and the floating radial head prosthesis. J. Shoulder Elbow Surg., 14(4):433-40, 2005.

Ring, D. Radial head fracture: open reduction-internal fixation or prosthetic replacement. J. Shoulder Elbow Surg., 20(2 Suppl.):S107-12, 2011.

Ring, D.; Quintero, J. \& Jupiter, J. B. Open reduction and internal fixation of fractures of the radial head. J. Bone Joint Surg. Am., 84(10):1811-5, 2002.

Roidis, N.; Stevanovic, M.; Martirosian, A.; Abbott, D. D.; McPherson, E. J. \& Itamura, J. M. A radiographic study of proximal radius anatomy with implications in radial head replacement. J. Shoulder Elbow Surg., 12(4):380-4, 2003.

Rosenblatt, Y.; Athwal, G. S. \& Faber, K. J. Current recommendations for the treatment of radial head fractures. Orthop. Clin. North Am., 39(2):173-85, 2008

Swieszkowski, W.; Skalski, K.; Pomianowski, S. \& Kedzior, K. The anatomic features of the radial head and their implication for prosthesis design. Clin. Biomech. (Bristol, Avon), 16(10):880-7, 2001.

\author{
Dirección para Correspondencia: \\ Edgardo S. Sanzana, M.D., Ph.D. \\ Profesor Titular \\ Departamento de Cirugía \\ Facultad de Medicina \\ Universidad de Concepción \\ Concepción \\ CHILE
}

Email: esanzana@udec.cl

Recibido : 07-12-2020

Aceptado: 10-01-2021 